\title{
ACOMPANHAMENTO DA ADAPTAÇÃO DE PRÓTESES AUDITIVAS EM CRIANÇAS SURDAS
}

\section{Evaluating the adaptation of hearing aids for hearing impaired children}

\author{
Bianca Pinheiro Lanzetta ${ }^{(1)}$, Silvana Frota ${ }^{(2)}$, Márcia Goldfeld ${ }^{(3)}$
}

\begin{abstract}
RESUMO
Objetivos: descrever as características audiológicas e sociais de crianças surdas e avaliar a incidência de retornos para acompanhamento no Programa de Saúde Auditiva. Métodos: foram analisados os prontuários de crianças que receberam as próteses auditivas pelo Programa de Saúde Auditiva, em Vila Velha - Espírito Santo. A população estudada foi constituída por 50 crianças, na faixa etária de zero a oito anos, de ambos os sexos, com diagnóstico de perda auditiva sensorioneural de grau leve a profundo. O protocolo de pesquisa foi preenchido a partir dos dados de prontuários para a obtenção das informações desejadas. Resultados: a solicitação de retorno pelo Serviço Social propiciou o comparecimento de quase da metade da população (44\%); os demais achados foram indicativos da associação entre o retorno para acompanhamento e a rotina escolar. Conclusões: o referido programa atinge predominantemente famílias com rendimento mensal entre um e dois salários mínimos; o diagnóstico da surdez ocorre entre dois e três anos de idade cronológica neste estudo; a época da primeira adaptação de próteses auditivas, aos seis anos de idade, é bastante tardia; o contato com os pais, por meio do Serviço Social, viabiliza o acompanhamento proposto, influenciado positivamente também pela rotina escolar.
\end{abstract}

DESCRITORES: Criança; Surdez; Auxiliares de Audição; Saúde Pública

\section{INTRODUÇÃO}

A integridade auditiva é fundamental para a aquisição e o desenvolvimento da linguagem, desempenhando papel importante na organização perceptual e na estrutruração das informações necessárias ao processo de aprendizagem e interação social do ser humano. Portanto, o desenvolvimento da audição e o da linguagem estão correlacionados ${ }^{1}$. O diagnóstico precoce da surdez, seguido de intervenção adequada, é determinante para o sucesso linguístico.

O Sistema Único de Saúde institui a Política Nacional de Atenção à Saúde Auditiva (Portaria

(1) Fonoaudióloga da Policlínica de Referência do Centro Universitário de Vila Velha, UVV, Vila Velha, ES, Brasil; Especialista em Audiologia pelo Conselho Federal de Fonoaudiologia; Mestre pela Universidade Veiga de Almeida.

(2) Fonoaudióloga; Professora Adjunta do Mestrado Profissionalizante em Fonoaudiologia da Universidade Veiga de Almeida, UVA, Rio de Janeiro, RJ, Brasil.

(3) Fonoaudióloga; Professora Adjunta do Mestrado Profissionalizante em Fonoaudiologia da Universidade Veiga de Almeida, UVA, Rio de Janeiro, RJ, Brasil.

Conflito de interesses: inexistente no. 2.073/2004) a partir da análise da magnitude social da deficiência auditiva e suas consequências na população brasileira. A implantação dessa Portaria possibilita o êxito da intervenção na deficiência auditiva, incentivando ações de promoção e prevenção em todos os níveis de atenção à saúde, por meio do trabalho de uma equipe multi e interprofissional especializada ${ }^{2}$.

Diante das consequências de um diagnóstico tardio da surdez, ressalta-se a importância da Triagem Auditiva Neonatal Universal (TANU), recomendada internacionalmente, independente da presença de indicadores de risco à deficiência auditiva. Segundo o Joint Comittee on Infant Hearing (2007), os fatores de risco são: preocupação dos pais com a audição e atraso no desenvolvimento da fala ou linguagem da criança; história familiar de deficiência auditiva permanente iniciada na infância; permanência em Unidade de Terapia Intensiva (UTI) neonatal por mais de cinco dias ou uso de ventilação mecânica prolongada; uso de medicamentos ototóxicos, hiperbilerrubinemia com exsanguíneo-transfusão; infecções congênitas (sífilis, toxoplasmose, rubéola, citomegalovírus e herpes); anomalias craniofaciais; 
síndromes que incluem a deficiência auditiva logo ao nascimento ou de início tardio; desordens neurodegenerativas; infecções pós-natais; traumatismo craniano com fratura do osso temporal, requerendo hospitalização e quimioterapia ${ }^{3}$.

Um estudo realizado em São Paulo sobre os aspectos diagnósticos e etiológicos da deficiência auditiva em crianças e adolescentes demonstra que a confirmação da surdez em fase adequada, referente à plasticidade neuronal da via auditiva (até dois anos), ocorre em apenas $13 \%$ dos casos, apesar da suspeita familiar presente em $56 \%$ das famílias nessa fase. A mãe é a primeira a suspeitar da deficiência auditiva da criança ( $72 \%$ dos casos), e o tempo entre a suspeita e o diagnóstico ultrapassa dois anos em $42 \%$ dos casos. A pesquisa não informa o intervalo transcorrido entre o diagnóstico, a protetização e o início do processo terapêutico ${ }^{4}$.

Em outra pesquisa realizada no Colorado (EUA), é possível observar que o diagnóstico precoce da surdez, favorecido pela realização da TANU, é acompanhado pelo processo de intervenção em tempo ideal. A distância entre o diagnóstico, que ocorre até o sexto mês de vida, e o início da terapia é de, no máximo, dois meses ${ }^{5}$.

Portanto, a TANU, por si só, não resulta em um melhor desenvolvimento infantil. A causa real de um desenvolvimento satisfatório está relacionada com a intervenção precoce, viabilizando a criança acesso à linguagem e à comunicação ${ }^{6}$.

Em um estudo no Rio Grande do Sul, Brasil, realizado com mães e/ou pais de crianças surdas, observa-se que a média de idade da suspeita é de 15 meses. A primeira consulta ao médico ou ao fonoaudiólogo ocorre aos 21 meses. O diagnóstico é aos 26 meses e a reabilitação inicia-se aos 41 meses. Esses dados ainda são preocupantes, quando comparados ao momento ideal à habilitação ou reabilitação auditiva ${ }^{7}$.

Pesquisas sobre a ansiedade dos pais de crianças surdas mostram semelhanças entre as famílias perante a confirmação diagnóstica da surdez, porém as expectativas são diferentes diante dessa nova realidade. Conclui-se que cabe ao fonoaudiólogo escutar e proporcionar respostas para cada dúvida dos familiares, dando-lhes suporte sempre que necessário ${ }^{8}$.

A perda auditiva tem como principal consequência a barreira na aquisição de línguas orais e auditivas. A falta de compreensão ou uma compreensão insuficiente da fala pode determinar à criança dificuldades linguísticas que influenciarão no seu desenvolvimento cognitivo, social e emocional ${ }^{9}$.

O papel do profissional no processo de habilitação ou reabilitação é, portanto, orientar os responsáveis e oferecer atendimento adequado visando à prevenção dessas consequências ${ }^{9}$.

O sucesso da adaptação de próteses auditivas depende do acompanhamento do paciente, para o uso efetivo da amplificação sonora ${ }^{10}$, que se torna viável por meio dos retornos periódicos ao Programa de Saúde Auditiva ${ }^{2}$.

O aconselhamento no processo terapêutico deve abranger questões fundamentais, como: motivar a integração e a adoção de posturas ativas quanto ao paciente, família e professores; promover a compreensão, a conscientização e a aceitação da deficiência auditiva e suas consequências, demonstrando atitudes que podem reduzir as dificuldades cotidianas; facilitar a aceitação e o uso efetivo das próteses auditivas ${ }^{11}$. Assim, o interesse, a participação e o seguimento das orientações tornam este processo eficaz.

O objetivo deste estudo é descrever as características audiológicas e sociais de crianças surdas e avaliar a incidência de retornos para acompanhamento no Programa de Saúde Auditiva do Estado do Espírito Santo.

\section{MÉTODOS}

Trata-se de um estudo descritivo, de caráter retrospectivo, realizado a partir do levantamento dos prontuários de 50 crianças surdas atendidas pelo convênio entre o Sistema Único de Saúde (SUS) e a Policlínica de Referência do Centro Universitário Vila Velha, durante o seu período inicial, de dezembro de 2005 a julho de 2008, no Estado do Espírito Santo.

Os prontuários foram analisados tendo em vista os critérios descritos a seguir: crianças, na faixa etária de um a oito anos, dos sexos feminino e masculino, deficientes auditivas e, consequentemente, apresentando indicação para o processo de seleção e adaptação de próteses auditivas.

Foram incluídas nesta pesquisa: crianças com a primeira prótese auditiva adquirida no Programa de Saúde Auditiva; crianças com idade cronológica de até oito anos; presença de surdez sensorioneural de grau leve, moderado, moderadamente severo, severo ou profundo; novos usuários de prótese auditiva, independentemente da categoria tecnológica do circuito de amplificação sonora.

Foram excluídas desta pesquisa: crianças sem diagnóstico definitivo de surdez sensorioneural; com diagnóstico confirmado de outros tipos de perda auditiva (condutiva ou mista); usuários de implante coclear; pacientes com múltiplas deficiências; portadores de perda auditiva unilateral, de neuropatia auditiva ou perda de audição progressiva; indivíduos com distúrbios graves de compor- 
tamento (agitação motora, déficit de atenção, entre outros) e rebaixamento cognitivo, inviabilizando a interação com o fonoaudiólogo.

O protocolo elaborado para a coleta e registro das informações foi composto pela caracterização da população estudada e pelos respectivos aspectos socioeconômico e familiar, composto por seis itens (Figura 1).
No primeiro item, constaram dados de identificação das crianças, como: inicial dos nomes, data de nascimento, idade de adaptação da prótese auditiva, número do prontuário, informante na anamnese (pai, mãe, ambos ou outros), rendimento mensal familiar ${ }^{12}$, anos de estudo da mãe ou responsável ${ }^{12}$ (nível de escolaridade), número de filhos e estado civil da mãe ou do responsável.

\section{Crianças surdas com prótese auditiva}

(0 - 8 anos)

\section{Identificação}

1.1.Nome:

1.2 Data de nascimento: 1

1.3 Idade de adaptação da prótese auditiva:

$1.4 \mathrm{~N}^{\circ}$ do prontuário:

1.5 Informante: 1. ( ) mãe 2.( ) pai 3.( ) mãe e pai 4. ( ) outros:

1.6 Classes de rendimento mensal familiar (IBGE, 2000):

1. ( ) Até 1 salário mínimo

2. ( ) Mais de 1 a 2 salários-mínimos

3. ( ) Mais de 3 a 5 salários-mínimos

4. ( ) Mais de 5 a 10 salários-mínimos

5. ( ) Mais de 10 a 20 salários-mínimos

6. ( ) Mais de 20 salários-mínimos

7. ( ) Sem rendimento

\subsection{Mãe - anos de estudos (IBGE, 2000):}

1. ( ) Sem instrução e menos de 1 ano

2. ( ) 1 a 3 anos

3. ( ) 4 a 7 anos

4. ( ) 8 a 10 anos

5. ( ) 11 a 14 anos

6. ( ) 15 anos ou mais

\subsection{Número de filhos:}

1. ( ) 1 a 2

2. ( ) 3 a 4

3. ( ) Acima de 4

\subsection{Estado civil da mãe (ou responsável):}

1. ( ) Solteiro

2. ( ) Casado

3. ( ) Separado

4. ( ) Viúvo 
1.10 A mãe (ou responsável) trabalha?

1.( ) Sim

2.( ) Não

\section{Idade do diagnóstico da surdez}

\subsection{Idade do diagnóstico da surdez}

1. ( ) Até 6 meses

2. ( ) Mais 6 meses -1 ano

3. ( ) Mais 1 ano -2 anos

4. ( ) Mais 2 anos -3 anos

5. ( ) A partir de 4 anos

\section{Grau da perda auditiva}

3.1 Caracterização da perda auditiva quanto ao grau da perda: (Média dos limiares de 500, 1000, $2000 \mathrm{~Hz}$ e $4000 \mathrm{~Hz})$.
1. ( ) Leve
2. ( ) Moderada
3. ( ) Moderadamente severa
4. ( ) Severa
5. ( ) Profunda

\section{Acompanhamento após o uso da prótese auditiva}

\subsection{Retorno para o acompanhamento}

1. ( ) Antes de 9 meses - espontâneo

2. ( ) Aos 9 meses - com solicitação da equipe

3. ( ) De 10 até 12 meses

4. ( ) Não retornaram mais de 12 meses

\section{Terapia fonoaudiológica}

\subsection{Terapia fonoaudiológica}

1. ( ) Sim, na Alta Complexidade

2. ( ) Sim, em outros locais

3. ( ) Não

\section{Rotina escolar}

\subsection{Frequenta escola?}

1. ( ) Sim, regular

2. ( ) Sim, especial

3. ( ) Sim, ambas

4. ( ) Não

Figura 1 - Protocolo de pesquisa 
No segundo item, foi caracterizada a idade do diagnóstico da surdez.

Verificou-se, no terceiro item, o grau da perda auditiva. Para tanto, foi utilizada a média aritmética entre os limiares de audibilidade, obtidos nas frequências sonoras de 500, 1000, $2000 \mathrm{~Hz}$ e $4000 \mathrm{~Hz}$. O grau da perda auditiva foi classificado em: leve (26 a $40 \mathrm{~dB} N A$ ), moderado (41 a $55 \mathrm{~dB}$ $\mathrm{NA}$ ), moderadamente severo (56 a $70 \mathrm{~dB} N A$ ), severo (71 a $90 \mathrm{~dB}$ NA) e profundo (a partir de $91 \mathrm{~dB}$ NA). Em casos de perdas auditivas assimétricas, foram considerados os limiares da melhor orelha ${ }^{13}$.

A seguir, pontuou-se o tempo de retorno para acompanhamento do uso das próteses auditivas na Alta Complexidade, quando foi realizada atenção diagnóstica ao deficiente auditivo ${ }^{2}$. Após a entrega das próteses auditivas, todas as famílias foram orientadas a retornar para acompanhamento após seis meses de uso da amplificação, ou antes, caso houvesse dúvidas, desconforto ou outros problemas durante o processo de adaptação.

Quando a criança não compareceu ao primeiro retorno marcado, o Serviço Social entrou em contato telefônico com o(s) reponsável(eis), aproximadamente nove meses após a aquisição das próteses auditivas. O retorno do paciente foi dividido em quatro possibilidades: antes dos nove meses, de forma espontânea; aos nove meses devido ao telefonema; após dez a doze meses; e na ausência de retorno, após as tentativas anteriores, constatou-se a evasão.

No item cinco, foi avaliado se a criança se encontrava ou não em terapia fonoaudiológica e em caso afirmativo, se o atendimento era realizado na Alta Complexidade ou em outro local.

$O$ item seis referiu-se à rotina escolar da criança. Foi verificado, entre os estudantes, qual o tipo de escola frequentada, isto é, regular e/ou especial.

Esta pesquisa foi submetida ao Comitê de Ética da Universidade Veiga de Almeida, Rio de Janeiro, e foi aprovada sob o no. 123/2008.

$\mathrm{Na}$ análise dos resultados obtidos, foram empregadas técnicas estatísticas descritiva e inferencial. $\mathrm{Na}$ análise inferencial, foi utilizado o teste não paramétrico qui-quadrado para verificar uma possível associação entre as variáveis estudadas. O nível de significância foi fixado em $5 \%$, assim o "p-valor" menor que 0,05 indica que existe relação estatisticamente significante entre as variáveis. Os programas computacionais utilizados foram: SPSS para Windows (versão 11.5) e Microsoft Word e Excel.

\section{RESULTADOS}

Quanto à faixa etária, a idade mínima foi 12 meses, sendo a média e a mediana correspondentes a seis anos, com desvio padrão igual a 1,9.

Para a variável sexo, houve predomínio do gênero masculino, representando $62 \%(n=31)$ da população estudada.

O informante mais frequente sobre o histórico da criança foi a sua própria mãe $(66 \%$ dos casos, $n=33)$, seguida pelo pai $(16 \%, n=8)$ ou ambos $(12 \%, n=6)$. A incidência de avós, irmãos e primo foi menor, correspondendo a $6 \%$ dos casos $(n=3)$.

Em relação ao rendimento mensal familiar, 37 famílias (74\% das participantes) apresentaram renda de um a dois salários-mínimos ao mês.

Quanto à escolaridade da mãe e/ou responsável pelo paciente, houve predomínio do nível fundamental incompleto (64\% dos casos, $n=32$ ). A presença de conclusão do ensino fundamental e do ensino médio foi observada, aproximadamente, em apenas 30\% ( $n=15)$ dessa população.

Considerando os achados sobre a quantidade de filhos, constatou-se maior frequência de mães com um a dois filhos $(74 \%, n=37)$, sendo minoria a presença de mães com três a quatro filhos.

De acordo com o estado civil da mãe ou responsável, verificou-se predomínio de pais casados (66\% dos casos, $n=33$ ). Em relação à presença de mães que trabalham fora de casa, foi possível observar que a maioria não possui emprego (58\%, $n=29$ ).

Quanto ao diagnóstico da surdez, em apenas três crianças ( $6 \%$ da população estudada) o tempo foi de até seis meses de idade; $10(20 \%)$ entre seis e 12 meses; oito (16\%), entre um ano e um ano e 11 meses; 19 (38\%) de dois a três anos; 10 (20\%) a partir de quatro anos.

Em relação ao grau da perda auditiva sensorioneural, prevaleceu o grau profundo, observado em 23 crianças (46\%). Apenas uma apresentou perda auditiva leve (2\%). Verificou-se grande maioria de perdas simétricas $(n=44 ; 88 \%)$.

A Tabela 1 demonstrou que a minoria das crianças compareceu espontaneamente ao acompanhamento proposto $(n=13,26 \%)$, número inferior ao de evasão do Programa ( $n=15 ; 30 \%)$, num período de 12 meses. A solicitação de retorno pelo Serviço Social propiciou o comparecimento de quase da metade população $(n=22 ; 44 \%)$.

$\mathrm{Na}$ Tabela 2 descreveu-se maior proporção estatística de crianças em acompanhamento fonoaudiológico. Foi possível observar que a maioria das crianças se encontrava em processo terapêutico (74\%). A fonoterapia é essencial para que haja 
de fato a habilitação ou reabilitação dos pacientes que receberam próteses auditivas, ou seja, a prótese representa apenas o primeiro passo desse processo.

A Tabela 3 determinou um predomínio de crianças no ensino regular. Dessas crianças, 90\% encontravam-se matriculadas em escolas regula- res e/ou especiais, resultado fundamental para o desenvolvimento comunicativo, além da integração social favorecida nessa condição.

Na Tabela 4, somente a variável escolaridade mostrou associação estatisticamente significante com o acompanhamento auditivo no Programa em questão.

Tabela 1 - Ocorrência de retornos da população estudada para o acompanhamento no Programa de Saúde Auditiva

\begin{tabular}{lcc}
\hline & \multicolumn{2}{c}{ Retorno para o acompanhamento } \\
\cline { 2 - 3 } & No absoluto & $\%$ \\
\hline Antes de 9 meses, espontâneo & 13 & 26,0 \\
Aos 9 meses, com solicitação da & 10 & 20,0 \\
equipe & 12 & 24,0 \\
De 10 até 12 meses, com solicitação & 15 & 30,0 \\
da equipe & 50 & 100,0 \\
Não retornaram mais de 12 meses & & \\
Total &
\end{tabular}

Tabela 2 - Distribuição das crianças segundo a realização de terapia fonoaudiológica

\begin{tabular}{lcc}
\hline & \multicolumn{2}{c}{ Terapia fonoaudiológica } \\
\cline { 2 - 3 } & № absoluto & $\%$ \\
\hline Sim, na Alta Complexidade & 15 & 30,0 \\
(Policlínica de Referência UVV) & 22 & 44,0 \\
Sim, em outros locais & 13 & 35,0 \\
Não & 50 & 100,0 \\
Total & &
\end{tabular}

Tabela 3 - Frequência de deficientes auditivos em escola regular e/ou especial

\begin{tabular}{lcc}
\hline & \multicolumn{2}{c}{ Frequenta escola } \\
\cline { 2 - 3 } & Frequência & $\%$ \\
\hline Sim, regular & 32 & 64,0 \\
Sim, especial & 4 & 8,0 \\
Sim, ambas & 9 & 18,0 \\
Não & 5 & 10,0 \\
Total & 50 & 100,0 \\
\hline
\end{tabular}


Tabela 4 - Relação entre as variáveis estudadas e o acompanhamento realizado no Programa de Saúde Auditiva

\begin{tabular}{|c|c|c|c|}
\hline Variáveis & Estatística de teste $-\chi^{2}$ & Valor-p & Resultado \\
\hline $\begin{array}{l}\text { Retorno para acompanhamento } x \\
\text { Classes de rendimento mensal } \\
\text { familiar }\end{array}$ & 7,457 & 0,194 & Não existe relação \\
\hline $\begin{array}{l}\text { Retorno para acompanhamento x } \\
\text { Anos de estudo da mãe }\end{array}$ & 15,297 & 0,367 & Não existe relação \\
\hline $\begin{array}{l}\text { Retorno para acompanhamento } \mathrm{x} \\
\text { № de filhos }\end{array}$ & 7,600 & 0,173 & Não existe relação \\
\hline $\begin{array}{l}\text { Retorno para acompanhamento x } \\
\text { Estado civil da mãe (ou responsável) }\end{array}$ & 6,972 & 0,637 & Não existe relação \\
\hline $\begin{array}{l}\text { Retorno para acompanhamento } x \\
\text { Condições de trabalho da mãe (ou } \\
\text { responsável) }\end{array}$ & 1,297 & 0,767 & Não existe relação \\
\hline $\begin{array}{l}\text { Retorno para acompanhamento } x \\
\text { Idade do diagnóstico de surdez }\end{array}$ & 11,986 & 0,400 & Não existe relação \\
\hline $\begin{array}{l}\text { Retorno para acompanhamento x } \\
\text { Caracterização da perda auditiva } \\
\text { quanto ao grau de perda }\end{array}$ & 11,649 & 0,453 & Não existe relação \\
\hline $\begin{array}{l}\text { Retorno para acompanhamento } \mathrm{x} \\
\text { Terapia fonoaudiológica }\end{array}$ & 3,826 & 0,737 & Não existe relação \\
\hline $\begin{array}{l}\text { Retorno para acompanhamento } x \\
\text { Frequenta escola }\end{array}$ & 13,974 & $0,043^{*}$ & Existe relação \\
\hline
\end{tabular}

Teste Qui-quadrado $\left(\chi^{2}\right)$

O resultado do teste indica que existe somente associação entre retorno para acompanhamento x rotina escolar, valor-p $<0,05$.

\section{DISCUSSÃO}

Diante do estudo retrospectivo realizado, das 360 crianças que entraram para avaliação audiológica no Programa de Saúde Auditiva, 117 crianças tiveram diagnóstico de surdez. Dessas, somente 50 foram analisadas, de acordo com os critérios de inclusão e exclusão desta pesquisa.

Foi observado que o Programa de Saúde Auditiva no Espírito Santo atinge parte da população de baixa renda familiar, o que corresponde ao perfil sociodemográfico e ao padrão de utilização de serviços de saúde dos usuários do SUS ${ }^{14}$.

Além disso, encontraram-se, neste estudo, famílias estruturadas com poucos filhos, pais casados e com mães que não trabalham, o que poderia influenciar na efetividade do uso da prótese auditiva nessas crianças.

No entanto, observou-se que o número de evasão é grande devido a fatores como: mudança de famílias para outros Estados, separações, prisões de pais, falecimento de criança, entre outros fatores.

A baixa natalidade verificada neste trabalho no Espírito Santo se justifica como uma tendência do Brasil. Um estudo focalizando mulheres de camadas populares de baixa renda, em Recife,
Pernambuco, procurou avaliar o planejamento familiar dessas mulheres. Com referência à política populacional, o País nunca utilizou o controle de natalidade de forma autoritária e totalitária do Estado quanto às decisões reprodutivas da população, a partir de interesses econômicos e de metas demográficas ${ }^{15}$.

Apesar de o País nunca ter utilizado políticas de controle de natalidade de forma autoritária, o planejamento familiar vem demonstrando baixa de natalidade ${ }^{16}$. Esse primeiro passo é essencial para a oferta de saúde e educação de qualidade para a população, no entanto, neste estudo, a estrutura familiar com poucos filhos ainda não foi um fator suficiente para promover o retorno das famílias ao Programa de Saúde Auditiva após o recebimento das próteses auditivas pelas crianças.

Além das características familiares, foram analisados alguns aspectos quanto às crianças surdas. Em relação à época do diagnóstico, houve predominância de 38\% ( $n=19)$ na idade cronológica entre dois e três anos.

Ao observar a idade, foi possível perceber que, no Brasil, ainda não se alcançou a idade ideal de diagnóstico da surdez, que deveria ser, no máximo, até os seis meses conforme a literatura ${ }^{5,6}$. 
Cabe enfatizar que a Policlínica de Referência de Referência do Centro Universitário Vila Velha é a única credenciada para o atendimento em saúde auditiva na Alta Complexidade no Estado do Espírito Santo. Seu atendimento à população iniciou-se em dezembro de 2005. Dessa forma, a maioria das crianças estudadas teve o diagnóstico da surdez anterior ao surgimento do Programa no Estado. Assim, com a implantação do programa, busca-se um diagnóstico e intervenção precoce da surdez.

Verificou-se, portanto, que a idade do diagnóstico da surdez das crianças estudadas no Espírito Santo está distante de referências internacionais ${ }^{5,6}$ e mais próxima da idade do diagnóstico encontrada em referências nacionais, em que o diagnóstico da surdez ocorreu aos 26 meses ${ }^{7}$.

Pontua-se que pouco mais da metade das crianças, mesmo com fatores de risco para surdez ${ }^{3}$, chega ao consultório antes de dois anos de idade, e esse tempo, na literatura, é considerado tardio demais. Esse fato reforça ainda a necessidade de se adotar uma postura intervencionista para evitar as sérias limitações que as crianças surdas terão em seu desenvolvimento auditivo, cognitivo, social e pessoal, com o intuito de minimizá-las, alcançado a idade ideal do diagnóstico da surdez ${ }^{4}$.

Em relação ao grau da perda auditiva, descreveu-se um maior percentual da perda auditiva de grau profundo de 23 (46\%) e curva audiométrica de configuração simétrica de 44 (88\%).

Cabe ressaltar que as consequências das perdas auditivas pré-linguísticas podem ser observadas conforme o grau específico dessa perda ${ }^{17}$. Mediante os aspectos descritos pelos autores, pode-se refletir que, a partir do perfil auditivo das crianças surdas, é possível pensar em estratégias mais eficazes de intervenção e reabilitação.

Para as características do retorno para acompanhamento das crianças surdas estudadas, foi observado que $13(26 \%)$ crianças tiveram retorno espontâneo até nove meses. O retorno com solicitação do Serviço Social aos nove meses ou de dez a doze meses ocorreu em 22 (44\%) crianças; e o não retorno, mesmo com solicitação e pressão da equipe, foi de 15 (30\%). Assim, o retorno espontâneo teve baixa ocorrência. Além disso, foi-observada a importância da intervenção da equipe (Serviço Social) para o retorno dessas crianças para acompanhamento.

No que diz respeito ao retorno para acompanhamento, estudos pontuam sua relevância no sentido de mapear a integralidade da assistência pelo acompanhamento da linha de cuidado, evitando-se a fragmentação da terapêutica instituída, priorizando a garantia dos cuidados necessários, o vínculo, a responsabilização com o usuário, a integralidade da assistência e o monitoramento contínuo dos resultados alcançados ${ }^{18}$.

Enfatiza-se que, de acordo com a literatura, o acompanhamento da criança deverá ocorrer nos primeiros anos de uso da prótese auditiva de forma trimestral e, após o segundo ano, no mínimo, semestralmente ${ }^{19}$, o que difere do proposto pela Portaria da Saúde Auditiva em relação às crianças entre três e quinze anos para as quais se propõe acompanhamento anual ${ }^{2}$.

Devido à escassez de estudos relacionados com o acompanhamento de crianças para a verificação da adaptação de próteses auditivas, foi feito um paralelo entre o retorno para acompanhamento do Programa de Saúde Auditiva para a adaptação da prótese auditiva e a TANU, considerando, os seguintes aspectos: faltas observadas em relação ao retorno para acompanhamento, e a dificuldade do retorno devido à distância do local onde são realizadas as avaliações ${ }^{20}$, uma vez que a Policlínica se localiza em Vila Velha e abrange uma demanda de todo o Estado do Espírito Santo.

Se os dados das crianças não estivessem organizados de forma a garantir a atuação do Serviço Social para crianças que não retornaram, o Programa de Saúde Auditiva teria perdido a oportunidade de acompanhar a adaptação da prótese auditiva de quase da metade das crianças selecionadas para o estudo.

No estudo sobre o processo de implantação de Programa de Saúde Auditiva em duas maternidades públicas, no estado do Espírito Santo, os autores descreveram que, para os recém-nascidos que falham no programa de triagem auditiva, apenas $53 \%$ retornaram para o reteste. Assim, foi constatada a evasão de $47 \%$, o que pode estar associado a fatores, como o grau de instrução de mães provenientes de maternidades públicas ${ }^{21}$.

Neste estudo a evasão foi de $30 \%$. Foi menor, quando comparada com outro estudo ${ }^{21}$. No entanto, os autores tiveram uma população estudada maior, o que pode ter interferido no resultado. A evasão observada neste estudo foi minimizada pela atuação do Serviço Social e pela busca do contato com as famílias.

Pontua-se o valor dessa atuação profissional dentro de uma equipe interdisciplinar na busca pela Saúde Auditiva. Todo esse processo só foi possível devido à organização e armazenamento de dados dos pacientes atendidos.

Existe um crescente interesse dos profissionais em redefinir os procedimentos para adaptação de próteses auditivas. Com os avanços tecnológicos das próteses auditivas, ocorre, consequentemente, necessidade de desenvolver métodos mais eficazes para avaliação da efetividade dessas próteses, 
com procedimentos capazes de prever o seu benefício auditivo na vida diária dos pacientes ${ }^{22}$.

Cabe ressaltar que, sobre o uso de próteses auditivas que avaliar e protetizar uma pessoa surda não é suficiente; existe a real necessidade de se acompanhar o sujeito protetizado para garantir uma boa adaptação e o uso efetivo da prótese auditiva ${ }^{10,19}$. Dessa forma, pontua-se a importância do acompanhamento do uso da prótese auditiva.

Também foi verificada a necessidade de fazer novas pesquisas relacionadas com o acompanhamento para realizar estudos comparativos e avaliar a adaptação da prótese auditiva em nosso país, em especial em crianças e idosos.

Além das características mencionadas, as crianças estão em acompanhamento fonoaudiológico, sendo $15(30 \%)$ delas na Alta Complexidade em Vila Velha - ES e 22 (44\%) em outros locais mais próximos de suas residências. Em relação à rotina escolar, ocorreu o predomínio estatístico de 32 (64\%) crianças estudantes do ensino regular.

Mesmo tendo apresentado uma maioria de crianças em atendimento fonoaudiológico, ainda $13(26 \%)$ não estão sendo acompanhadas. Analisando, em contexto mais amplo, a população geral que procura o Serviço de Saúde Auditiva, a partir dos relatos de pais ao Serviço Social, acredita-se que a falta de acompanhamento fonoaudiológico seja ainda significativamente maior. A partir desses dados, pode-se pensar em uma população sem intervenção fonoaudiológica que não busca o serviço e não está protetizada. Esse fato remete à necessidade da realização de estudos acerca do acompanhamento fonoaudiológico dessa população.

Em relação ao acompanhamento fonoaudiológico, os autores pontuam a importância do diagnóstico e intervenção da surdez, apontando que a sua ocorrência tardia pode provocar dificuldades linguísticas que irão ocasionar prejuízos ao desenvolvimento cognitivo, social e emocional criança. Descrevem também a importância da participação da família nesse processo ${ }^{1,4-7,9,11}$. Dessa forma, o acompanhamento para a adaptação de próteses auditivas e o desenvolvimento infantil tornam-se indissociáveis.

Ao analisar as relações das variáveis estudadas com o retorno para acompanhamento, observou-se a relação entre retorno e rotina escolar ( $p$-valor de 0,043). Evidencia-se a importância da rotina escolar como influenciadora do retorno das crianças surdas para o acompanhamento na Alta Complexidade, visto que a melhora da percepção auditiva pode ser considerada como um fator fundamental para o processo de aprendizagem escolar. Estudos demonstram que a maioria dos pais deseja que seus filhos estudem em classes regulares, outros pais acreditam que as classes especiais são mais adequadas e outros preferem que os filhos estudem complementarmente em classe regular e especial $^{8}$. Contudo, nesta pesquisa, estar no ensino regular foi um fator determinante para o retorno para o acompanhamento.

Os dados obtidos demonstraram pontos que possibilitam uma atuação reflexiva sobre o uso da prótese auditiva. A partir desses dados, podem-se formar estratégias e rever atendimentos, acolhimento, orientações e encaminhamentos diante das crianças surdas e suas famílias, para aprimorar a atuação clínica fonoaudiológica e interdisciplinar em busca da Saúde Auditiva no Estado do Espírito Santo.

\section{CONCLUSÃO}

O Programa de Saúde Auditiva do Espírito Santo atinge predominantemente famílias com rendimento mensal entre um e dois salários mínimos; o diagnóstico da surdez ocorre entre dois e três anos de idade cronológica neste estudo; a época da primeira adaptação de próteses auditivas, aos seis anos de idade, é bastante tardia; o contato com os pais, por meio do Serviço Social, viabiliza o acompanhamento proposto, influenciado positivamente também pela rotina escolar. 


\section{ABSTRACT}

Purpose: to describe the compliance and attitudes of hearing-impaired children towards the treatment and support offered by the Hearing Health Program, a public health endeavor, and assessing patients' returns for follow-ups. Methods: participants consisted of fifty children aged from 0 to 8 years, with a diagnosis of mild to severe sensorineural hearing loss. The children received the hearing aids from the Hearing Health Program, in Vila Velha, in the state of Espírito Santo, Brazil. The research protocol was completed using both medical records and a socio-economical profile survey of the affected children, including the behavioral adaptation as for hearing aids. Results: the requirement of patient return for follow-ups facilitated the attendance of nearly half the population (44\%); results indicate that there seems to be an association between compliance with the program and school routine. Conclusion: the aforementioned program mostly affects families with a monthly income from one to two minimum wages. Hearing loss is diagnosed between the chronological ages of two and three years in this study. The first adaptation with hearing aids at the age of six is quite late. The contact with the parents promoted by the social workers facilitates the proposed evaluation. School attendance also proved to have a positive influence on the evaluation.

KEYWORDS: Children; Hearing Loss; Hearing Aids; Public Health

\section{REFERÊNCIAS}

1. Gatto $\mathrm{Cl}$, Tochetto TM. Deficiência auditiva infantil implicações e soluções. Rev. CEFAC. 2007; 9(1):110-5. http://dx.doi.org/10.1590/ S1516-18462007000100014

2. Brasil. Portaria no. 2.073, de 28 de setembro de 2004. Institui a Política Nacional de Atenção à Saúde Auditiva. Diário Oficial [da] República Federativa do Brasil. Ministério da Saúde, Gabinete Ministerial, Brasília; 2004.

3. Joint Committee on Infant Hearing. American Academy of Pediatrics. Year 2007 position statement: principles and guidelines for early hearing detection and intervention programs. Pediatrics. 2007; 120(4):898-921.

4. Nóbrega M, Weckx LL, Juliano Y. Study of the hearing loss in children and adolescents, comparing the periods of 1990-1994 and 1994-2000. Int J Pediatr Otorhinolaryngol. 2005; 69(6):829-38.

5. Yoshinaga-Itano C. Early intervention after universal neonatal hearing screening: impact on outcomes. Ment Retard Dev Disabil Res Rev. 2003; 9(4):252-66. http://dx.doi.org/10.1002/mrdd.10088

6. Yoshinaga-Itano $\mathrm{C}$. Levels of evidence: universal newborn hearing screening (UNHS) and early hearing detection and intervention systems (EHDI). J Commun Disord. 2004; 37(5):451-65.

7. Heck F, Raymann BCW. Tempo de decorrido entre a suspeita da surdez, a primeira ida ao médico e/ou fonoaudiólogo, o diagnóstico e o início da reabilitação com crianças surdas. J Bras Fonoaudiol. 2003; 4(16):175-85.
8. Boscolo CC, Santos TMM. A deficiência auditiva e a família: sentimentos e expectativas de um grupo de pais de crianças com deficiência da audição. Dist Comun. 2005; 17(1):69-75.

9. Goldfeld, M. Atendimento fonoaudiológico para surdos com enfoque bilíngüe e interacionista. In: Frota S, Goldfeld M, organizadores. O ouvir e o falar: enfoques em audiologia e surdez. São Paulo: AM3artes; 2006. p. 282-320.

10. Ribas A, Rosa M, Dante G, Martins-Bassetto J. SOS prótese auditiva: relato de um estudo de caso realizado na clínica de fonoaudiologia da UTP. Ciência e Cultura. 2006; 38:9-18.

11. Souza MCF, Wieselberg MB. Aconselhamento em audiologia. In: Lopes Filho $\mathrm{O}$, organizador. Tratado de fonoaudiologia. Ribeirão Preto: Tecmedd; 2005. p.555-71.

12. IBGE: Instituto Brasileiro de Geografia e Estatística. Censo demográfico 2000: resultados da amostra. [acesso em 3 abr 2008] Disponível em: http://www. sidra.ibge.gov.br

13. Momensohn-Santos TMM, Russo IP, Borgianni L. Interpretação dos resultados da avaliação audiológica In: Momensohn-Santos TM, Russo ICP, organizadores. Prática da audiologia clínica. 5.ed. São Paulo: Cortez; 2007. p. 291-310.

14. Ribeiro MCSA, Barata RB, Almeida MF, Silva ZP. Perfil sociodemográfico e padrão de utilização de serviços de saúde para usuários e não-usuários do SUS-PNAD 2003. Ciênc Saúde Coletiva. 2006; 11(4):1011-22.

15. Fernandes MFM. Mulher, família e reprodução: um estudo de caso sobre o planejamento familiar 
em periferia de Recife. Cad Saúde Pública. 2003; 19(Supl2):253-61.

16. Costa AM, Guilhem D, Silver LD. Planejamento familiar: a autonomia das mulheres sob questão. Rev Bras Saúde Matern Infant. 2006; 6(1):75-84. 17. Lim SYC, Simser J. Auditory-verbal therapy for children with hearing impairment. Ann Acad Med. 2005; 34(4):307-12.

18. Malta DC, Cecilio LCO, Merhy EE, Franco TB, Jorge AO, Costa MA. Perspectivas da regulação na saúde suplementar diante dos modelos assistenciais. Ciênc Saúde Coletiva. 2004; 9(2):4333-444. 19. Almeida K, Santos TMM. Seleção e adaptação de próteses auditivas em crianças. In: Almeida K, lorio N. Próteses auditivas, fundamentos teóricos e aplicações clínicas. 2. ed. São Paulo: Lovise; 2003. p. 357-84.

20. Fichino SN, Meyer EP, Lewis DR. Acompanhamento audiológico de crianças com indicadores de perda auditiva. Dist Comun. 2000; 11(2):313-33.

21. Barreira-Nielsen C, Futuro Neto HA, Gattaz G. Processo de implantação de Programa da Saúde Auditiva em duas maternidades públicas. Rev Soc Bras Fonoaudiol. 2007; 12(2):99-105.

22. Almeida K, Taguchi CK. Utilização do questionário na auto-avaliação do beneficio das próteses auditivas. Pró-Fono. 2004; 16(1):101-10.

RECEBIDO EM: 17/03/2009

ACEITO EM: 08/12/2009

Endereço para correspondência:

Bianca Pinheiro Lanzetta

Rua Mercúrio, s/n

Vila Velha - ES

CEP: 29102-623

E-mail: bianca.lanzetta@terra.com.br 